\title{
Consideraciones para la atención odontológica durante la pandemia de la COVID-19
}

\author{
Considerations for dental care \\ during the COVID-19 pandemic
}

Aarón Josué Ramos Ramos ${ }^{1 a}$

Isabel Del Rosario Ayca Castro ${ }^{2 b c}$

Wender Williams Condori Quispe ${ }^{3 \mathrm{bd}}$

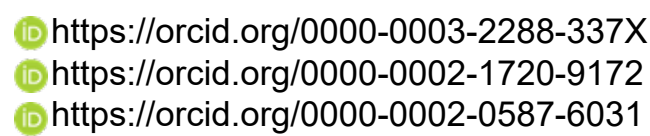

Correspondencia: ar2021071733@virtual.upt.pe

\section{Resumen}

La pandemia causada por el virus SARS-CoV-2 ha demostrado ser de fácil transmisión, de pacientes portadores a pacientes clínicamente sanos, donde la principal vía de contagio es el contacto directo. Teniendo conocimiento de dicha información y considerando que la profesión odontológica ya era una de las que mayor riesgo de exposición a contagios tiene, la COVID-19 ha incrementado mucho más ese riesgo laboral en el área odontológica. Por tal motivo, si el uso de medidas de bioseguridad era un requisito indispensable para la atención odontológica, es de mucha mayor importancia reforzar los conocimientos de bioseguridad; así mismo, realizar una correcta valoración del paciente, en cuanto a sintomatología, para poder realizar un correcto diagnóstico y permitir al odontólogo proceder con la electividad de tratamientos odontólogicos. Para la elaboración del presente artículo se realizó la búsqueda de información relacionada con la atención odontológica en el contexto de la pandemia de la COVID-19; dándose mayor énfasis a las medidas de prevención para evitar el contagio de la COVID-19, a las urgencias y emergencias odontológicas y a la electividad de los tratamientos odontológicos. Concluyéndose que para evitar el contagio de la COVID-19, la principal barrera de protección es el uso adecuado de las medidas de bioseguridad, realizar una correcta anamnesis del paciente para proceder con la valoración y, dependiendo del resultado, proceder con la mejor opción de tratamiento, considerándose que en la actual pandemia de la COVID-19 se recomienda la atención a urgencias y emergencias odontológicas.

Palabras clave: COVID-19, bioseguridad, personal de odontología

\section{Abstract}

The pandemic caused by the SARS-CoV-2 virus has proven to be easily transmitted, from carrier patients to clinically healthy patients, where the main route of infection is direct contact. Having knowledge of this information and considering that the dental profession was already one of those with the highest risk of exposure to contagions, COVID-19 has increased, in the dental area, that occupational risk much more. For this reason, it is that if the use of biosafety measures was an essential requirement for dental care, it is of much greater importance to reinforce biosafety knowledge; likewise, make a correct assessment of the patient, in terms of symptoms, in order to make a correct diagnosis and allow the dentist to proceed with the choice of dental treatments. For the preparation of this article, a search was carried out for information related to dental care in the context of the COVID-19 pandemic; giving greater emphasis to prevention measures to avoid the spread of COVID-19, dental emergencies, and the electivity of dental treatments. Concluding that to avoid the spread of COVID-19 the main protection barrier is the proper use of biosafety measures, taking a correct anamnesis of the patient to proceed with the assessment and depending on the result proceed with the best treatment option, considering that in the current COVID-19 pandemic, care is recommended for dental emergencies.

Keywords: COVID-19, biosafety, dental staff

\footnotetext{
${ }^{1}$ Universidad Privada de Tacna. Escuela de Posgrado. Tacna, Perú

2 Sociedad Peruana de Odontopediatría

3 Sociedad Peruana de Ortodoncia

a Maestrando en Investigación Científica e Innovación. Cirujano Dentista

${ }^{\text {b }}$ Maestro en Odontoestomatología

${ }^{c}$ Especialista en Odontopediatría

d Especialista en Ortodoncia y Ortopedia Maxilar
} 


\section{Introducción}

La COVID-19, la cual hace referencia a coronavirus disease o enfermedad del coronavirus, fue catalogada como pandemia por la Organización Mundial de la Salud (OMS). Dicha enfermedad va causando muchas pérdidas humanas a nivel global debido a la fácil transmisibilidad entre pacientes portadores del SARS-CoV-2 y personas clínicamente sanas; considerándose dentro del grupo de portadores a los asintomáticos como la principal problemática puesto que no presentan ningún tipo de síntoma y aun así tienen la capacidad de transmitir la enfermedad. ${ }^{1}$ En la actualidad, la mayoría de países han paralizado casi por completo sus actividades diarias con la finalidad de frenar el avance de la COVID-19; lo cual ha conllevado, en países de bajos recursos económicos, una serie de efectos secundarios negativos tales como hambre, miseria, deuda y desempleo. ${ }^{2}$

Durante la actual pandemia de la COVID-19 se ha determinado que la principal vía de contagio es el contacto directo de persona a persona (con portadores del virus SARS-CoV-2), la cual se da mediante tos, estornudos, pequeñas gotas de saliva y mediante fluidos respiratorios, ${ }^{3}$ así como también por contacto indirecto por medio de las manos, objetos o superficies contaminadas. ${ }^{4}$ Teniendo en consideración dichas vías de contagio es que los profesionales del área de salud están considerados dentro del grupo de riesgo a contagiarse y más aún los odontólogos, considerados como miembros de una de las profesiones con altísimo riesgo laboral, ${ }^{5}$ debido a que la mayoría de los tratamientos odontológicos requieren de una actividad cara a cara con el paciente, contacto directo con la saliva, sangre u otros fluidos, uso de materiales punzocortantes o afilados. A esto se debe sumar que el uso de la turbina de aire, también conocida como pieza de alta, ultrasonidos o la acción de escupir, etc., son la principal fuente generadora de aerosoles, además, considerando que se ha encontrado que el SARS-CoV-2 puede detectarse en la saliva de pacientes infectados, esto solo incrementa el riesgo de contagio hacia el odontólogo. ${ }^{6,7}$ Se debe considerar que se ha informado que el SARS-CoV-2 puede permanecer en superficies inanimadas hasta 9 días, ${ }^{8}$ por lo que es de entender que el ambiente del consultorio odontológico sería un punto para la propagación de la enfermedad de la COVID-19.

Por tal motivo, si el uso de medidas de bioseguridad era ya un requisito para la atención odontológica, en el contexto de la pandemia de la COVID-19 ya es indispensable reforzar y enfatizar la aplicación de las normas básicas de bioseguridad con el uso de equipos de protección personal, lavado de manos, adecuación del consultorio, selección de los casos según gravedad y necesidad de tratamiento, etc. ${ }^{9-12}$ En el Perú, conforme el número de casos diagnosticados de la COVID-19 fueron incrementándose, al mismo tiempo, el Ministerio de Salud (MINSA), mediante la Directiva Sanitaria No100-MINSA-2020-DGIESP, dispone la aplicación obligatoria, en los establecimientos de salud donde se realice actividad odontológica, una serie de medidas de bioseguridad para disminuir el riesgo de infección por COVID-19 durante la atención estomatológica. ${ }^{13}$ De igual manera, el Colegio Odontológico del Perú (COP) elabora el Protocolo de bioseguridad para el cirujano dentista durante y post pandemia COVID-19, en donde se determina la priorización de la atención de solo urgencias y emergencias, ${ }^{14-17}$ aplicando nuevas medidas en la práctica estomatológica; tales como el triaje telefónico o la telemedicina. ${ }^{18,19}$ Todas estas recomendaciones formarán parte de la "nueva normalidad", marcando un precedente en la odontología. ${ }^{20}$

Por tal motivo, la búsqueda de información realizada en este estudio es de importancia, puesto que la profesión odontológica, si ya antes de la pandemia era considerada de riesgo, actualmente, durante la pandemia de la COVID-19, es considerada como uno de los mayores riesgos debido al contacto directo con los pacientes y sus fluidos bucales, los cuales son vistos como la principal fuente de propagación del virus SARS-CoV-2 en pacientes positivos, tanto sintomáticos como asintomáticos. Por ende, es de suma importancia saber cuáles son las principales recomendaciones dadas por los autores en cuanto a la atención dental durante la pandemia de la COVID-19. Así mismo, se plantea el siguiente objetivo general: Describir las principales recomendaciones de protección personal en la atención dental en casos de urgencias o emergencias odontológicas durante la pandemia de la COVID-19. De la misma forma se formulan los siguientes objetivos específicos: a) Describir las recomendaciones que brindan los autores de los artículos revisados para el uso del equipo de protección personal en la atención dental; b) Precisar las 
recomendaciones de los artículos revisados sobre la atención de urgencias y emergencias odontológicas; y c) Precisar las recomendaciones que brindan los autores consultados sobre la electividad de tratamientos odontológicos según la emergencia sanitaria de la COVID-19.

Este estudio, de revisión de literatura, se sustenta en la importancia y necesidad de dar mayores alcances en los conocimientos referidos a las medidas necesarias para evitar el contagio y propagación de la COVID-19 en la atención odontológica, puesto que, como se menciona, esta profesión es considerada dentro de las más riesgosas por las actividades realizadas en los tratamientos. Se busca, de la misma forma, actualizar alcances y conceptos de acuerdo a la electividad y necesidad de tratamientos requeridos por los pacientes hacia una atención odontológica, puesto que los alcances científicos, con respecto a la semiología de la enfermedad de la COVID-19, se van actualizando constantemente conforme va avanzando la situación de la actual pandemia.

\section{Selección de fuentes de información}

El presente artículo es un artículo de revisión. El cual se caracteriza por no ser original, sino más bien por ser la recopilación de bibliografía más relevante publicada anterior a este estudio y sobre un tema específico. ${ }^{21}$ Se realizó una revisión de la literatura en la base de datos SciELO mediante la siguiente estrategia de búsqueda: periodo de publicación (enero del 2020 a abril del 2021); idiomas de publicación (español); términos de búsqueda: "(coronavirus) AND (dental)". Se seleccionaron artículos de revisión de literatura y ensayos de revistas de acceso abierto; el criterio de exclusión fue que aquellos estudios que no estén relacionados con la COVID-19 y atención dental fueran eliminados de la selección.

La búsqueda inicial arrojó 93 estudios, de los cuales 73 fueron eliminados porque no eran relevantes para el tema de investigación. Los 20 artículos restantes se revisaron para confirmar si cumplían con el criterio de inclusión relacionado a la COVID-19 y las recomendaciones para la atención odontológica; y, finalmente, se seleccionaron 10 estudios para un análisis integral. En la Figura 1 se grafica este proceso.

\section{Figura 1}

Diagrama de flujo de selección de artículos científicos

\section{Identificación}

Elegibilidad

\section{Inclusión}

-93 artículos científicos en Scielo

-.---> 73 artículos eliminados por título

-20 artículos científicos incluidos por título

•-.--> 10 artículos eliminados por criterio de inclusión

-10 artículos científicos en la revisión

Los 10 artículos incluidos en el análisis son pertinentes al tema de atención odontológica y la COVID-19, y tienen mención de las principales recomendaciones de medidas de prevención de la COVID-19. En la Tabla 1 se presentan las principales características de los 10 estudios que, en opinión del autor, presentan los resultados más relevantes con respecto al objetivo de esta revisión (recomendaciones para la atención odontológica durante la pandemia de la COVID-19) y proponen las recomendaciones para la práctica odontológica. 
Tabla 1

Estudios relacionados a odontología durante la pandemia de la COVID-19

\begin{tabular}{|c|c|c|c|c|}
\hline $\begin{array}{l}\text { Autor o } \\
\text { autores }\end{array}$ & Objetivo & $\begin{array}{l}\text { Diseño, tipo de } \\
\text { estudio }\end{array}$ & $\begin{array}{c}\text { Población o } \\
\text { unidades de } \\
\text { estudio }\end{array}$ & Resultados principales \\
\hline $\begin{array}{l}\text { Bustillos Torres } \\
\text { W, Bueno Bravo } \\
\text { ZS. }\end{array}$ & $\begin{array}{l}\text { Dar a conocer la } \\
\text { importancia de la } \\
\text { bioseguridad en } \\
\text { odontología en tiempos } \\
\text { de coronavirus }\end{array}$ & Artículo de revisión & $\begin{array}{l}3 \text { estudios } \\
\text { realizados en } \\
\text { América }\end{array}$ & $\begin{array}{l}\text { Existirán modificaciones } \\
\text { en bioseguridad después } \\
\text { de la COVID-19, de } \\
\text { las cuales, algunas } \\
\text { serán verificadas por } \\
\text { organismos del área a } \\
\text { nivel mundial como CDC, } \\
\text { ADA, etc., las cuales } \\
\text { deberán ser socializadas } \\
\text { en la consulta privada, } \\
\text { estatal y en las } \\
\text { universidades. }\end{array}$ \\
\hline $\begin{array}{l}\text { Morales } \\
\text { Navarro D. }\end{array}$ & $\begin{array}{l}\text { Esclarecer los } \\
\text { elementos relacionados } \\
\text { con la COVID-19 y } \\
\text { las medidas a tener } \\
\text { en cuenta para la } \\
\text { prevención del contagio } \\
\text { del personal de salud } \\
\text { del área estomatológica }\end{array}$ & Ensayo & - & $\begin{array}{l}\text { Es de mucha importancia } \\
\text { que el personal de salud, } \\
\text { dentro de ellos los del } \\
\text { área estomatológica, } \\
\text { y el público en general } \\
\text { conozcan de la COVID-19 } \\
\text { con la finalidad de tomar } \\
\text { acciones coordinadas, } \\
\text { oportunas y efectivas } \\
\text { que ayuden a prevenir } \\
\text { la propagación de la } \\
\text { enfermedad. }\end{array}$ \\
\hline $\begin{array}{l}\text { Quincho- } \\
\text { Rosales } \\
\text { DA, Castro- } \\
\text { Rodríguez } \\
\text { Y, Grados- } \\
\text { Palomino S. }\end{array}$ & $\begin{array}{l}\text { Describir el desarrollo } \\
\text { de la pandemia de la } \\
\text { COVID-19 en el Perú } \\
\text { y brindar algunas } \\
\text { consideraciones para la } \\
\text { salud bucal durante la } \\
\text { emergencia sanitaria, } \\
\text { así como mencionar } \\
\text { los principales retos } \\
\text { en la consulta estatal y } \\
\text { privada en odontología. }\end{array}$ & Ensayo & - & $\begin{array}{l}\text { La profesión odontológica } \\
\text { es la de mayor riesgo } \\
\text { durante la pandemia } \\
\text { por COVID-19; a pesar } \\
\text { de ello, el odontólogo } \\
\text { debe cumplir su rol } \\
\text { como personal de salud } \\
\text { siguiendo los protocolos } \\
\text { de bioseguridad } \\
\text { establecidos por las } \\
\text { instituciones de salud. }\end{array}$ \\
\hline $\begin{array}{l}\text { Aylwin MI, } \\
\text { Richaud H.C., } \\
\text { Moreno Y.Y. }\end{array}$ & $\begin{array}{l}\text { Dar a conocer las } \\
\text { orientaciones y } \\
\text { medidas de prevención } \\
\text { contra la COVID-19 en } \\
\text { odontología basados en } \\
\text { la evidencia. }\end{array}$ & Ensayo & - & $\begin{array}{l}\text { Con la finalidad de } \\
\text { prevenir la transmisión } \\
\text { de agentes microbianos } \\
\text { y virus, se debe aplicar } \\
\text { medidas de precaución } \\
\text { universales para todo } \\
\text { paciente; se trate de } \\
\text { contagiado o no. La } \\
\text { atención en pacientes con } \\
\text { sintomatología debe ser } \\
\text { coordinada con manejo } \\
\text { médico y evitar el uso de } \\
\text { material rotatorio. }\end{array}$ \\
\hline
\end{tabular}




\begin{tabular}{|c|c|c|c|c|}
\hline $\begin{array}{l}\text { Maestre J.D., } \\
\text { Rosabal L.C. }\end{array}$ & $\begin{array}{l}\text { Identificar las } \\
\text { medidas de } \\
\text { prevención y control } \\
\text { de la COVID-19 } \\
\text { después de una } \\
\text { eventual reapertura } \\
\text { de los servicios } \\
\text { o consultorios } \\
\text { odontológicos. }\end{array}$ & Ensayo & - & $\begin{array}{l}\text { Se concluye que el } \\
\text { regreso a la nueva } \\
\text { normalidad obligó a } \\
\text { elevar las medidas } \\
\text { de protección con la } \\
\text { finalidad de evitar el } \\
\text { contagio de infecciones } \\
\text { en la consulta } \\
\text { estomatológica, } \\
\text { asegurando no solo a los } \\
\text { pacientes, sino también } \\
\text { al personal de salud y } \\
\text { sus familiares. }\end{array}$ \\
\hline Guiñez C.M. & $\begin{array}{l}\text { Dar a conocer } \\
\text { el impacto de } \\
\text { la COVID-19 } \\
\text { en el mundo, } \\
\text { las implicancias } \\
\text { y medidas de } \\
\text { prevención en la } \\
\text { práctica dental y } \\
\text { las consecuencias } \\
\text { psicológicas en los } \\
\text { pacientes. }\end{array}$ & $\begin{array}{l}\text { Artículo de revisión } \\
\text { sistemática }\end{array}$ & $\begin{array}{l}16 \text { artículos } \\
\text { relacionados con } \\
\text { la COVID-19, salud } \\
\text { pública y odontología } \\
\text { en las bases de } \\
\text { datos Medline } \\
\text { y ClinicalKey; } \\
\text { excluyendo aquellos } \\
\text { estudios en animales } \\
\text { y literatura gris. }\end{array}$ & $\begin{array}{l}\text { La mayoría de } \\
\text { procedimientos } \\
\text { odontológicos producen } \\
\text { la generación de } \\
\text { aerosoles, por este } \\
\text { motivo se deben } \\
\text { tomar medidas de } \\
\text { protección, selección } \\
\text { de pacientes a tratar y } \\
\text { tipos de tratamientos } \\
\text { a desarrollar, con la } \\
\text { finalidad de disminuir la } \\
\text { posibilidad de infecciones } \\
\text { en el personal de salud } \\
\text { y pacientes. En la } \\
\text { situación de pandemia } \\
\text { de la COVID-19 evaluar } \\
\text { la posibilidad de entregar } \\
\text { soporte psicológico } \\
\text { a los pacientes, en } \\
\text { especial a aquellos que } \\
\text { padecen de patologías } \\
\text { psicosomáticas. }\end{array}$ \\
\hline $\begin{array}{l}\text { Herrera- } \\
\text { Plasencia P.M., } \\
\text { Enoki-Miñano E., } \\
\text { Ruiz-Barrueto M. } \\
\text { y Ruiz-Barrueto } \\
\text { A. }\end{array}$ & $\begin{array}{l}\text { Identificar cuáles } \\
\text { son los principales } \\
\text { riesgos, vías de } \\
\text { transmisión y } \\
\text { medidas preventivas } \\
\text { a tomar en } \\
\text { cuenta frente a la } \\
\text { COVID-19. }\end{array}$ & $\begin{array}{l}\text { Artículo de revisión } \\
\text { de literatura }\end{array}$ & $\begin{array}{l}50 \text { artículos } \\
\text { relacionados con } \\
\text { los descriptores } \\
\text { "coronavirus", } \\
\text { "COVID-19", "dental", } \\
\text { "dentistry", "oral", } \\
\text { "stomatology" y } \\
\text { "aerosol" }\end{array}$ & $\begin{array}{l}\text { La profesión } \\
\text { odontológica es la que } \\
\text { tiene un mayor riesgo } \\
\text { potencial a contraer } \\
\text { infecciones; por lo tanto, } \\
\text { es de suma importancia } \\
\text { conocer el agente causal } \\
\text { y la enfermedad, lo que } \\
\text { ayudará a reducir la } \\
\text { posibilidad de contagio. } \\
\text { El odontólogo debe } \\
\text { de considerar a todos } \\
\text { los pacientes como } \\
\text { sospechosos de portar la } \\
\text { COVID-19. }\end{array}$ \\
\hline
\end{tabular}




\begin{tabular}{|c|c|c|c|c|}
\hline $\begin{array}{l}\text { Sepúlveda V.C., } \\
\text { Secchi A.A., y } \\
\text { Donoso-Hofer F. }\end{array}$ & $\begin{array}{l}\text { Describir, mediante el } \\
\text { análisis de literatura } \\
\text { actualizada, las } \\
\text { recomendaciones } \\
\text { que tienen por } \\
\text { finalidad disminuir el } \\
\text { contagio frente a la } \\
\text { inminente exposición } \\
\text { directa a pacientes } \\
\text { sospechosos o que } \\
\text { pudiesen presentar } \\
\text { COVID-19. }\end{array}$ & $\begin{array}{l}\text { Artículo de revisión } \\
\text { de literatura }\end{array}$ & $\begin{array}{l}18 \text { referencias } \\
\text { bibliográficas citadas } \\
\text { en el artículo }\end{array}$ & $\begin{array}{l}\text { En el caso de las } \\
\text { urgencias odontológicas } \\
\text { que no puedan ser } \\
\text { diferidas, el odontólogo } \\
\text { deberá tomar en } \\
\text { consideración las } \\
\text { recomendaciones dadas } \\
\text { en el presente estudio } \\
\text { (lavado de manos, } \\
\text { desinfección y equipo } \\
\text { de protección personal) } \\
\text { solo así se estaría } \\
\text { reduciendo al mínimo } \\
\text { las probabilidades } \\
\text { de contagio entre el } \\
\text { operador y el paciente. }\end{array}$ \\
\hline $\begin{array}{l}\text { Boin B.C. y } \\
\text { Melián R.A. }\end{array}$ & $\begin{array}{l}\text { Mostrar las medidas } \\
\text { de protección } \\
\text { exitosas optadas por } \\
\text { otros países para } \\
\text { evitar la propagación } \\
\text { de la COVID-19. }\end{array}$ & $\begin{array}{l}\text { Ensayo de opinión } \\
\text { propia }\end{array}$ & & $\begin{array}{l}\text { Debido a que una gran } \\
\text { cantidad de pacientes } \\
\text { son asintomáticos, esto } \\
\text { significaría un alto riesgo } \\
\text { para el odontólogo; por } \\
\text { lo tanto, es de suma } \\
\text { importancia la aplicación } \\
\text { de medidas básicas } \\
\text { de protección para los } \\
\text { pacientes y el personal } \\
\text { de salud. }\end{array}$ \\
\hline Araya S.C. & $\begin{array}{l}\text { Dar a conocer las } \\
\text { consideraciones } \\
\text { necesarias para } \\
\text { la atención } \\
\text { de urgencias } \\
\text { odontológicas y } \\
\text { las medidas de } \\
\text { prevención para } \\
\text { evitar el contagio de } \\
\text { la COVID-19 }\end{array}$ & $\begin{array}{l}\text { Ensayo de opinión } \\
\text { propia }\end{array}$ & & $\begin{array}{l}\text { La atención odontológica } \\
\text { en el contexto de la } \\
\text { COVID-19 debe de ser } \\
\text { electiva, con la finalidad } \\
\text { de disminuir contagios } \\
\text { y exposiciones. El } \\
\text { correcto examen y } \\
\text { determinación de una } \\
\text { urgencia odontológica, } \\
\text { acompañado de } \\
\text { una anamnesis } \\
\text { epidemiológica, es } \\
\text { fundamental para la } \\
\text { elección de tratamiento } \\
\text { o derivación para una } \\
\text { atención hospitalaria. }\end{array}$ \\
\hline
\end{tabular}

\section{Método de análisis de las fuentes de información}

Los 10 artículos seleccionados por criterio de inclusión son detallados a continuación, en la Tabla 2, con respecto a la relación de los objetivos de estudio y la información pertinente correspondiente a cada unidad de análisis. 
Tabla 2

Estudios analizados y su relación con los objetivos planteados

\section{Título}

Importancia de la bioseguridad en odontología, en tiempo de coronavirus

Acciones del personal de salud del área estomatológica en relación a la COVID-19

Consideraciones sobre la atención estomatológica en el Perú durante la pandemia por la COVID-19

Guía para el trabajo clínico en odontología durante la pandemia por SARS-CoV-2 en el hospital clínico Dra. Eloísa Díaz

\section{Medidas de prevención y control} de la COVID-19 en estomatología: "la nueva normalidad"

Impacto de la COVID-19 (SARSCoV-2) a nivel mundial, implicancias y medidas preventivas en la práctica dental y sus consecuencias psicológicas en los pacientes

Riesgos, contaminación y prevención frente a la COVID-19 en el quehacer odontológico: una revisión

\section{Objetivos del estudio}

Referido a medidas de prevención

Referido a medidas de

Referido a valoración del paciente

Referido a urgencias y emergencias

Referido a valoración del paciente

Referido a medidas de prevención

Referido a medidas de prevención

Referido a medidas de prevención paciente prevención

\section{Información pertinente}

Da a conocer sobre los riesgos a los que está expuesto el odontólogo y la importancia de la bioseguridad en su actividad diaria; así mismo, menciona la importancia del esquema de vacunación en profesionales de la salud.

Menciona cuáles son las principales medidas para evitar la infección asociada a la atención médica, detallando los implementos de protección personal necesarios para la atención del paciente (sospechoso o confirmado).

Da a conocer cuál es el tipo de atención que puede recibir el paciente según su diagnóstico, incluyendo recomendaciones para su atención.

Explica de manera resumida las urgencias odontológicas, el tratamiento y el instrumental a utilizar para la resolución del caso

Describe la clasificación de pacientes (caso no sospechoso, sospechoso o probable o no confirmado, confirmado de COVID-19 y recuperado) y los tratamientos odontológicos electivos según sea el caso.

Identifica cuáles son las medidas de prevención y control de la COVID-19 en el desarrollo de actividades odontológicas, desde la adecuación del ambiente hasta el desarrollo de tratamientos.

El estudio describe medidas de prevención, así como también refiere la importancia del equipo de protección personal como protocolo y la adecuación del consultorio para la atención odontológica.

Referido a valoración del Menciona la estandarización de una serie de preguntas que permitan valorar de cierta manera el registro de antecedentes epidemiológicos con la finalidad de poder determinar qué tan factible sea la realización de algún tratamiento odontológico.

En el presente estudio se describe cuáles son las vías de contagio, manifestaciones clínicas y medidas de prevención para evitar el contagio de la COVID-19, dando énfasis a las barreras de protección personal y a la minimización del uso de instrumentos generadores de aerosoles. 
Consideraciones para la atención de urgencia odontológica y medidas preventivas para la COVID-19 (SARS-CoV-2)

La atención odontológica a pacientes con COVID-19 positivo ¿Qué hacer ante una urgencia?

Consideraciones en la atención odontológica de urgencia de contexto de la COVID-19 (SARSCoV-2)
Referido a urgencias y emergencias

Referido a urgencias y emergencias

Referido a urgencias y emergencias
Indica, mediante una tabla, la clasificación de la American Dental Association (ADA) para las emergencias y urgencias dentales y algunas recomendaciones a seguir para el tratamiento de estas.

Describe la importancia de las medidas de bioseguridad y de la anamnesis epidemiológica en los pacientes a tratar, así como también menciona las acciones a tomar cuando el personal odontológico estuvo en contacto con pacientes COVID-19 positivos o sospechosos. Informa de las principales consideraciones generales, previas, durante y posteriores al tratamiento de urgencias odontológicas para reducir el contagio de la COVID-19 entre el operador odontológico y el paciente.

\section{Generalidades de la pandemia de la COVID-19}

En diciembre del año 2019, en la ciudad de Wuhan, provincia de Hubei (China), surge un nuevo tipo de coronavirus, el cual es nombrado, temporalmente, por la Organización Mundial de la Salud (OMS), como "nuevo coronavirus 2019: 2019-nCoV"; posteriormente, conforme se fue diseminando el virus pasó a llamarse SARS-CoV-2, agente causal de la enfermedad de la COVID-19. Dicha enfermedad es considerada como emergencia de salud pública por la OMS el 23 de enero del 2020, para luego ser considerada, por la misma institución, como pandemia el 11 de marzo., ${ }^{9,22}$ En el Perú, el 23 de marzo del 2020, la COVID-19 obliga al gobierno a declarar la emergencia sanitaria, la cual, en un inicio, fue establecida para 90 días, que incluían medidas restrictivas de movilización social para evitar el contagio de la enfermedad. ${ }^{10}$ Actualmente, 4 de julio del 2021, según el reporte del Ministerio de Salud, en la página del Gobierno del Perú, se registra un total de 2065113 casos totales, indicando que la letalidad de la COVID-19 es de 9.36 \% en la población; así mismo, se informa que la vacunación tiene un avance de $64.6 \%$ con vacuna completa, lo cual es un buen indicador para combatir la propagación de la enfermedad y evitar complicaciones que terminen en el fallecimiento de peruanos contagiados. ${ }^{23}$

Una de las principales preocupaciones de las instituciones de salud, nacionales e internacionales, es la capacidad de transmisión que, comparada a otros virus, es mucho más rápida; además, cualquier persona es susceptible de ser contagiada. Los infectados por la COVID-19 se pueden clasificar en dos grandes grupos: sintomáticos (aquellos que desarrollan los principales síntomas de la enfermedad; fiebre, tos, congestión nasal, rinorrea, dolor de garganta, afectación pulmonar, cefalea, náusea, vómito, diarrea) ${ }^{12,20}$ y asintomáticos (aquellos que no muestran ningún tipo de afectación en su organismo). Ambos tipos de pacientes son potencialmente agentes transmisores de la enfermedad; por lo tanto, causa mayor preocupación aquel paciente asintomático que padezca de alguna enfermedad dental y acuda al consultorio odontológico requiriendo tratamiento, puesto que las formas de transmisión conocidas hasta la fecha son mediante transmisión directa (estornudos, pequeñas gotas de saliva, mediante la generación de aerosoles y contacto de las mucosas nasal, oral y ocular) y transmisión indirecta (al tocar superficies inanimadas contaminadas y luego tocarse la boca, nariz o los ojos)..$^{24,25}$ Por lo tanto, dichas formas de transmisión son el principal fundamento para que el profesional odontológico refuerce sus conocimientos sobre la aplicación de medidas de bioseguridad para evitar el contagio por COVID-19 en la atención dental.

\section{Medidas de prevención}

En cuanto a las medidas de prevención descritas en los artículos analizados se describe un antes, durante y después de la atención odontológica. Con la finalidad de reducir al mínimo el riesgo de contagio de la COVID-19, en las Tablas 3, 4 y 5 se describe las recomendaciones de medidas de prevención. 


\section{Tabla 3}

Medidas de prevención para evitar el contagio de la COVID-19 antes de la atención dental

\section{Antes de la atención dental}

Limpieza de la suela

del zapato

Higiene de las manos

Valoración del

paciente

\section{De la sala de espera}

En la entrada deberá contarse con un tapete humedecido en una solución de hipoclorito de sodio al $1.5 \%$ para que los pacientes que ingresen limpien la suela del calzado.

Todo paciente que acuda al consultorio odontológico deberá ingresar con su mascarilla y haber realizado un lavado de manos con agua y jabón de al menos 30 segundos o utilizando alcohol en gel de $70 \%$. Ambos métodos están diseñados para la eliminación de suciedad, materia orgánica y microbiota transitoria.

Se debe realizar la toma de temperatura (sin contacto) y la aplicación de un cuestionario

1. ¿Ha experimentado fiebre en los últimos 14 días? ¿Usa antipiréticos?

2. ¿Ha experimentado algún problema respiratorio reciente, como tos o dificultad respiratoria en los últimos 14 días?

3. ¿Ha viajado o visitado lugares con casos de COVID-19?

4. ¿Ha estado en contacto con pacientes confirmados con COVID-19 en los últimos 14 días?

5. ¿Ha estado en contacto con personas que hayan viajado a lugares con casos de COVID-19 o en zonas con casos recientes de fiebre o problemas respiratorios en los últimos 14 días?

6. ¿Ha estado en contacto con personas con fiebre o problemas respiratorios en los últimos 14 días?

7. ¿Ha participado recientemente en cualquier reunión o estado en contacto cercano con muchas personas desconocidas?

- Si el paciente responde "sí", en cualquiera de las preguntas, y tiene una temperatura menor a $37.3^{\circ} \mathrm{C}$ se puede referir el tratamiento y recomendar una autocuarentena.

- Si el paciente responde "sí", en cualquiera de las preguntas, y tiene una temperatura mayor o igual a $37.3^{\circ} \mathrm{C}$ debe ser enviado a cuarentena y reportarlo al centro de salud más cercano.

- Si el paciente responde "no" en cualquiera de las preguntas y tiene una temperatura menor a $37.3^{\circ} \mathrm{C}$ se puede tratar con las medidas de protección, pero si no es emergencia diferir.

- Si el paciente responde "no" en cualquiera de las preguntas y tiene una temperatura mayor o igual a $37.3^{\circ} \mathrm{C}$ se debe evaluar la causa de fiebre, sino debe ser derivado al centro de salud más cercano.

Se bloquearán asientos en la sala de espera con la finalidad de mantener el correcto distanciamiento social $(1.5 \mathrm{~m})$, los pacientes deben ser citados en horarios diferentes y evitar acompañantes.

Adecuar un lugar en el cual las pertenencias del paciente sean almacenadas 


\section{Tabla 4}

Medidas de prevención para evitar el contagio de COVID-19 durante la atención dental

\begin{tabular}{|c|c|}
\hline \multicolumn{2}{|r|}{ Durante la atención dental } \\
\hline Lavado de manos & $\begin{array}{l}\text { Será un prerrequisito, para el odontólogo, realizar el lavado de manos, el cual debe de } \\
\text { efectuarse " } 2 \text { antes y } 3 \text { después" } \\
\text { 1. Antes de examinar al paciente } \\
\text { 2. Antes de iniciar un procedimiento } \\
\text { 3. Después de tocar alguna superficie o equipo sin desinfección } \\
\text { 4. Después de tocar al paciente } \\
\text { 5. Después de tocar la mucosa oral, piel dañada, sangre, fluidos corporales, } \\
\text { secreciones y excretas. }\end{array}$ \\
\hline $\begin{array}{l}\text { Equipo de } \\
\text { protección personal } \\
\text { (EPP) }\end{array}$ & $\begin{array}{l}\text { Consiste en el uso de barreras físicas para evitar el contagio de algún tipo de infección; } \\
\text { se debe realizar el lavado de manos antes de colocarse el EPP: } \\
\text { - Guantes (doble guante) } \\
\text { - Respiradores N95 o FFP2; su uso no debe exceder las } 4 \text { horas } \\
\text { - Mandil } \\
\text { - Gorro } \\
\text { - Lentes de protección } \\
\text { - Botas } \\
\text { - Máscara facial }\end{array}$ \\
\hline Colutorios & $\begin{array}{l}\text { El virus SARS-CoV-2 es susceptible a la peroxidación, por lo tanto, es recomendable } \\
\text { que el paciente utilice los siguientes tipos de colutorios: peróxido de hidrógeno al } 1 \% \text { - } \\
1.5 \% \text { (durante } 1 \text { minuto), yodopovidona al } 0.2 \% \text { o cloruro de cetilpiridino (CPC) al } 0.05 \\
\%-0.1 \% \text { (durante } 30 \text { segundos). }\end{array}$ \\
\hline Goma dique & $\begin{array}{l}\text { Se recomienda el uso de goma dique en los tratamientos en los cuales es necesaria la } \\
\text { utilización de equipo rotatorio, puesto que su uso demostró la disminución de un } 70 \text { \% } \\
\text { de la producción de salpicaduras o aerosol. }\end{array}$ \\
\hline $\begin{array}{l}\text { Succión de alta } \\
\text { potencia }\end{array}$ & $\begin{array}{l}\text { Adicional al uso de la goma dique, se menciona la utilización de una aspiración de alta } \\
\text { potencia para el aerosol y succión regular para las salpicaduras }\end{array}$ \\
\hline Trabajo a 4 manos & $\begin{array}{l}\text { Se recomienda, para todos los procedimientos, la técnica de trabajo conjunto "a cuatro } \\
\text { manos", puesto que disminuye el tiempo de atención y hace más eficiente el trabajo. }\end{array}$ \\
\hline $\begin{array}{l}\text { Instrumental } \\
\text { generador de } \\
\text { aerosoles }\end{array}$ & $\begin{array}{l}\text { Se recomienda el uso de agentes químicos o manuales antes de instrumental rotatorio, } \\
\text { ultrasonido o la jeringa triple; debido a que estos contribuyen a la formación de aero- } \\
\text { soles. Si es necesaria la utilización de pieza de mano de alta velocidad, se recomienda } \\
\text { aquellas que posean un sistema de antirretorno. }\end{array}$ \\
\hline $\begin{array}{l}\text { De la sala de } \\
\text { operaciones }\end{array}$ & $\begin{array}{l}\text { Se recomienda tener una buena ventilación, abriendo la ventana por lo menos } 3 \text { veces al } \\
\text { día, durante } 30 \text { minutos. } \\
\text { También se menciona que es ideal la atención odontológica en salas de procedimiento } \\
\text { con presión negativa. }\end{array}$ \\
\hline
\end{tabular}




\section{Tabla 5}

Medidas de prevención para evitar el contagio de COVID-19 después de la atención dental

\section{Después de la atención dental}

\begin{tabular}{|c|c|}
\hline Desinfección & $\begin{array}{l}\text { Después de la atención dental se recomienda desinfectar las superficies de trabajo y de } \\
\text { contacto de personal y paciente, siempre con guantes y con un trapo húmedo de hipoclorito de } \\
\text { sodio ( } 30 \mathrm{ml} \text { de cloro en } 1 \text { litro de agua) } \\
\text { Si es necesario se puede utilizar radiación UV, dos veces al día durante } 30 \text { minutos. } \\
\text { También se puede emplear el uso de un bactericida en aerosol para sanear la sala de } \\
\text { operaciones. } \\
\text { Las principales áreas a desinfectar son: } \\
\text { - Sillón dental } \\
\text { - Salivero } \\
\text { - Lámpara } \\
\text { - Brazos de mesa de trabajo } \\
\text { - Manijas de las puertas } \\
\text { - Sillas de operadores } \\
\text { - Escritorios } \\
\text { Si se utilizó instrumental rotatorio, este se debe desinfectar con alcohol de } 70 \text { \% para } \\
\text { posteriormente ser esterilizado. }\end{array}$ \\
\hline Ventilación & $\begin{array}{l}\text { Se recomienda que la sala de operaciones sea ventilada por lo menos } 15 \text { a } 30 \text { minutos en } \\
\text { aquellos procedimientos sin generación de aerosoles; y en caso se haya generado aerosoles, } \\
\text { el tiempo mínimo es de } 30 \text { minutos. }\end{array}$ \\
\hline $\begin{array}{l}\text { Manipulación de } \\
\text { desechos }\end{array}$ & $\begin{array}{l}\text { Los desechos orgánicos y el material descartable utilizados en la atención de pacientes debe } \\
\text { estar en bolsas de doble capa y deberá rotularse indicando contaminación de material por } \\
\text { coronavirus. }\end{array}$ \\
\hline $\begin{array}{l}\text { Lavado de } \\
\text { manos }\end{array}$ & $\begin{array}{l}\text { Una vez terminada la atención dental y despachado el paciente, el operador y el personal } \\
\text { deberá lavarse las manos y cara. }\end{array}$ \\
\hline
\end{tabular}

\section{Urgencias y emergencias}

En Perú, desde el 16 de marzo del 2020, solo se limita la atención odontológica en aquellos casos que sean considerados como urgencias o emergencias estomatológicas y que, en la mayoría de casos, requieren del uso de la pieza de alta velocidad; en consecuencia, es recomendable programar la cita de estos pacientes en la última hora de la agenda diaria.

Son consideradas urgencias aquellas patologías odontológicas que tengan como principal síntoma el dolor, las cuales por lo menos deben ser evaluadas por el profesional odontológico para un adecuado tratamiento; para ellos podemos mencionar en la Tabla 6 aquellas urgencias estomatológicas más comunes, el tratamiento y el instrumental necesario. 


\section{Tabla 6}

Urgencia estomatológica, tratamiento e instrumental recomendado

\begin{tabular}{|c|c|c|}
\hline Entidad & Tratamiento & $\begin{array}{c}\text { Instrumental generador de } \\
\text { aerosol }\end{array}$ \\
\hline Pulpitis & \multirow[t]{2}{*}{$\begin{array}{l}\text { Apertura cameral, } \\
\text { desbridamiento pulpar, drenaje }\end{array}$} & \multirow[t]{2}{*}{$\begin{array}{l}\text { Turbina de aire (pieza de mano } \\
\text { de alta velocidad) }\end{array}$} \\
\hline Absceso periapical & & \\
\hline Pericoronaritis & $\begin{array}{l}\text { Exodoncia compleja, } \\
\text { desbridamiento, drenaje }\end{array}$ & $\begin{array}{l}\text { Turbina de aire (pieza de mano } \\
\text { de alta velocidad), bisturí } \\
\text { piezoeléctrico o ultrasónico }\end{array}$ \\
\hline Complicaciones en exodoncia & Exodoncia a colgajo/completa & $\begin{array}{l}\text { Turbina de aire (pieza de mano } \\
\text { de alta velocidad), bisturí } \\
\text { piezoeléctrico o ultrasónico }\end{array}$ \\
\hline $\begin{array}{l}\text { Prótesis fija con pilares } \\
\text { dentarios con pulpitis, } \\
\text { absceso periapical, etc. }\end{array}$ & $\begin{array}{l}\text { Retiro de prótesis, tratamiento } \\
\text { de piezas según la patología }\end{array}$ & $\begin{array}{l}\text { Turbina de aire (pieza de mano } \\
\text { de alta velocidad) }\end{array}$ \\
\hline Periimplantitis & Retiro de implante & $\begin{array}{l}\text { Turbina de aire (pieza de mano } \\
\text { de alta velocidad), bisturí } \\
\text { piezoeléctrico o ultrasónico, } \\
\text { motor de implante }\end{array}$ \\
\hline
\end{tabular}

Además de estas urgencias estomatológicas, la American Dental Association (ADA) cataloga, dentro de las urgencias dentales, aquellos procedimientos tales como toma de biopsia de tejidos anormales, cementación de prótesis o provisionales (unitarias o plurales) que se hayan extraviado y exista daño en mucosa y/o encía.

Por otro lado, las emergencias según la ADA son clasificadas como todos aquellos cuadros patológicos que sean una potencial amenaza a la vida del paciente y requieran de un tratamiento inmediato para su resolución; para tal caso se puede mencionar las siguientes emergencias:

- Hemorragias profusas difíciles de controlar.

- Infección y aumento de volumen difuso extra-intraoral con posible compromiso de vía aérea.

- Trauma maxilofacial con posible compromiso de vía aérea.

- Electividad de tratamientos odontológicos.

Los estudios analizados mencionan la importancia de la atención de las urgencias estomatológicas de manera oportuna, con la finalidad de evitar complicaciones de estas y que después evolucione a una emergencia estomatológica poniendo en riesgo la vida del paciente. También se menciona, en lo posible, minimizar el uso de instrumental generador de aerosol; priorizando, para el cuadro de pulpitis, la medicación analgésica antiinflamatoria. Si el caso no evoluciona favorablemente se recurrirá al uso de instrumental no rotatorio (curetas de dentina); si a pesar de esto no se logra acceder a la cámara pulpar, se recomienda el instrumental de baja velocidad, considerando como última opción la utilización de la turbina de aire (pieza de alta velocidad). Otra opción mencionada es el uso de agentes químico-mecánicos los cuales disminuyen la percepción del dolor por parte del paciente; sin embargo, su principal desventaja es el incremento de tiempo operatorio, que llega a ser 2.5 veces más comparado al uso de la turbina de aire, y su costo económico adicional. Para el caso de las emergencias que requieran sutura, los estudios recomiendan el 
uso de materiales reabsorbibles, con la finalidad de evitar un segundo contacto con el paciente después del tratamiento estomatológico.

\section{Electividad de tratamientos odontológicos}

La electividad del paciente, según sea la urgencia o emergencia, dependerá de una correcta anamnesis del paciente; si bien es cierto se debe de considerar a todo paciente como potencialmente infeccioso y a todo fluido como potencialmente contaminante, esto solo refuerza el principio de universalidad en bioseguridad. Por ende, es de importancia elegir bien cuándo tratar al paciente en el contexto de la pandemia de la COVID-19, por eso es que a continuación (Tabla 7) se menciona el procedimiento recomendado a seguir según la valoración del paciente.

\section{Valoración del paciente}

Caso no sospechoso de
infección SARS-CoV-2

Caso sospechoso o probable de infección SARS-CoV-2, no confirmado

Caso confirmado de COVID-19

Caso COVID-19 recuperado

\section{Descripción}

Asintomático o con diagnóstico negativo (24 horas previa a la cita odontológica).

Aquellos pacientes que presenten al menos 2 síntomas o cualquier persona con infección respiratoria aguda (IRA).

Caso probable: aquel paciente que tenga al menos 1 síntoma y que ha sido expuesto a un paciente confirmado con COVID-19.

Caso sospechoso en el cual la prueba PCR para COVID-19 resultó positiva.

Un paciente recuperado se considera portador del SARS-CoV-2 hasta 30 días después de confirmar su recuperación con una prueba de laboratorio.

\section{Indicación}

Puede ser atendido inmediatamente después del triaje inicial.

Se puede realizar tratamientos de urgencias, minimizando procedimientos que generen aerosoles (PGA), en caso de ser necesario, en los PGA se deberá utilizar goma dique.

Si el paciente pertenece a un grupo de alto riesgo de morbilidad o mortalidad, solo se deberá realizar tratamientos de urgencia o emergencia.

Posponer toda atención electiva.

Solo tratar urgencias o emergencias minimizando los PGA.

Si después de una prueba de descarte el paciente resultara negativo, se esperará hasta 15 días para realizar tratamientos estomatológicos.

Si el paciente resultara positivo, se esperará hasta 30 días después del periodo de cuarentena.

Posponer o diferir todo tratamiento que no sea de urgencia.

Si el caso es de urgencia, se deberá coordinar con personal médico para la atención multidisciplinaria.

Se sugiere ser derivado a emergencia médica para atención.

Se atenderá al paciente si ha estado asintomático desde 14 a 30 días posterior al inicio de síntomas o de que fuera diagnosticado. Se puede realizar tratamientos de urgencia y tratamientos minimizando los PGA. 


\section{Conclusiones}

Las recomendaciones de prevención para evitar el contagio por SARS-CoV-2 en la atención dental van enfocadas en la correcta aplicación de medidas de bioseguridad; puesto que los profesionales de odontología son los de mayor riesgo a contagio debido a las actividades que realizan, como el contacto directo con el paciente, con una aproximación a la cavidad bucal (considerada vía de transmisión directa), contacto con la saliva (la cual es considerada como un medio de transmisión de la COVID-19) y la producción de aerosoles debido al uso de la turbina de aire (pieza de alta velocidad) solo incrementan más aún la posibilidad de contagio de la COVID-19.

Por tal motivo es que se recomienda el uso adecuado del equipo de protección personal, la adecuación de la sala de operaciones priorizando una buena ventilación, realizar una correcta anamnesis del paciente para su valoración y dependiendo del resultado proceder con la mejor opción de tratamiento. Considerar que en contexto de la pandemia de la COVID-19 se recomienda realizar solo procedimientos que estén catalogados como urgencias o emergencias; el odontólogo deberá diferenciar la necesidad y la electividad del tratamiento según la valoración del paciente. Para el caso de las urgencias se recomienda minimizar, en lo posible, aquellos procedimientos generadores de aerosoles y para las emergencias la derivación a atención médica especializada y multidisciplinar.

\section{Referencias}

1. Sacsaquispe-Contreras S. NUEVO CORONAVIRUS 2019 (COVID-19): Consejos para el odontólogo. Rev Estomatológica Hered [Internet]. 2020 Mar 13;30(1):5-6. Available from: https://revistas.upch.edu. pe/index.php/REH/article/view/3671

2. Caduff C. Qué salió mal: el coronavirus y el mundo después del pare total. Rev Econ Inst. 2021;23(44):14369.

3. González-Quintanilla D, Santos-López M. Medidas Preventivas y Consideraciones para la Práctica de Cirugía Oral durante COVID-19. Int J Odontostomatol [Internet]. 2020 Sep;14(3):338-41. Available from: http://www.scielo.cl/scielo.php?script=sci_arttext\&pid=S0718-381X2020000300338\&lng=en\&nrm=iso\& tlng=en

4. Figueiredo MC, Armas-Vega A del C, Martínez AM, Agudelo-Suárez AA, Carletto-Korber FPM, Quiñónez GBM, et al. Covid-19 y la odontología latinoamericana para pacientes con necesidades especiales. Odontoestomatologia [Internet]. 2021 May 17;23(37):1-7. Available from: https://www.odon.edu.uy/ojs/ index.php/ode/article/view/342

5. Gispert Abreu EA, Chaple Gil AM. Gestión de la atención de salud bucal en tiempos de la COVID-19. Rev Cuba Estomatol. 2020;57(4):e3442.

6. Pérez N, Díaz-Reissner C, Adorno C, Fretes V, Escobar J, Gamarra J, et al. Evaluation of the dispersion of aerosols produced during the use of the dental turbine using the ejector of the equipment and/or extraoral suction. Exploratory study. Rev salud publica del Paraguay [Internet]. 2021 Jun 30;11(1):28-32. Available from: http://scielo.iics.una.py/scielo.php?script=sci_arttext\&pid=S2307-33492021000100028\&lng=es\&n rm=iso\&tlng=es

7. Venegas-Arques MC, Rojas-García CP, Cataldo-Saavedra YA, Jiménez-Gomez PF, Arqués-Vergara VI, Martinez B. Contaminación Bacteriana del Aerosol Dental con y sin Uso de una Cúpula de Acrílico en un Paciente en Pandemia COVID-19. Int J Odontostomatol [Internet]. 2021 Mar;15(1):14-22. Available from: http://www.scielo.cl/scielo.php?script=sci_arttext\&pid=S0718-381X2021000100014\&lng=en\&nrm $=$ iso\&tlng=en 
8. Suárez SalgadoS, Campuzano R, Dona Vidale M, Garrido Cisneros E, Gimenez Miniello T. Recomendaciones para prevención y control de infecciones por SARS-CoV-2 en odontología. Odontol (Habana) [Internet]. 2020 Jul 1;22(2):5-32. Available from: http://revistadigital.uce.edu.ec/index.php/odontologia/article/ view/2211/2163

9. Bustillos Torrez W, Bueno Bravo ZS. Importance of Biosecurity in Dentistry, in times of coronaviruses. Rev salud publica del Paraguay [Internet]. 2021 Jun 30;11(1):80-6. Available from: http://scielo.iics.una.py/ scielo.php?script=sci_arttext\&pid=S2307-33492021000100080\&lng=es\&nrm=iso\&tlng=es

10. Quincho-RosalesDA, Castro-RodríguezY, Sixto-GradosP.Consideraciones sobrela atención estomatológica en el Perú durante la pandemia por la COVID-19. Rev Cuba Estomatol2 [Internet]. 2020;57(3):e3315. Available from: http://www.revestomatologia.sld.cu/index.php/est/article/view/3315

11. Herrera-Plasencia PM, Enoki-Miñano ER, Ruiz-Barrueto MA. Riesgos, contaminación y prevención frente al COVID-19 en el quehacer odontológico: una revisión. Rev Salud Pública [Internet]. 2020 Sep 1;22(5):16. Available from: https://revistas.unal.edu.co/index.php/revsaludpublica/article/view/86065

12. Guiñez-Coelho M. Impacto del COVID-19 (SARS-CoV-2) a Nivel Mundial, Implicancias y Medidas Preventivas en la Práctica Dental y sus Consecuencias Psicológicas en los Pacientes. Int J Odontostomatol [Internet]. 2020 Sep;14(3):271-8. Available from: http://www.scielo.cl/scielo.php?script=sci_arttext\&pid=S0718$381 \times 2020000300271 \&$ Ing=en\&nrm=iso\&tlng=en

13. Ministerio de Salud. Manejo de la atención estomatológica en el contexto de la pandemia por COVID-19. Directiva sanitaria No100/MINSA/2020/DGIESP. 2020. p. 1-44.

14. Sepúlveda VC, Sechi AA, Donoso-Hofer F. Consideraciones en la atención odontológica de urgencia en contexto de coronavirus COVID-19 (SARS-CoV-2). Int J Odontostomat. 2020;14(3):279-84.

15. Araya-Salas $\mathrm{C}$. Consideraciones para la Atención de Urgencia Odontológica y Medidas Preventivas para COVID-19 (SARS-CoV 2). Int J Odontostomatol [Internet]. 2020 Sep;14(3):268-70. Available from: http:// www.scielo.cl/scielo.php?script=sci_arttext\&pid=S0718-381X2020000300268\&lng=en\&nrm=iso\&tlng=en

16. Boin-Bakit C, Melián-Rivas A. La atención Odontológica a Pacientes COVID-19 Positivo ¿Qué hacer ante una Urgencia? Int J Odontostomatol [Internet]. 2020 Sep;14(3):321-4. Available from: http://www.scielo.cl/ scielo.php?script=sci_arttext\&pid=S0718-381X2020000300321\&lng=en\&nrm=iso\&tlng=en

17. Velásquez-González A, Sepúlveda-Verdugo C, Ortuño-Borroto D, Barrientos-Morales C. Recomendaciones para Resolución de Urgencias Odontológicas en Atención Primaria de Salud Durante la Pandemia de SARS-CoV-2. Int J Odontostomatol [Internet]. 2020 Dec;14(4):548-54. Available from: http://www.scielo.cl/ scielo.php?script=sci_arttext\&pid=S0718-381X2020000400548\&lng=en\&nrm=iso\&tIng=en

18. Colegio Odontológico del Perú. Protocolo de bioseguridad para el cirujano dentista durante y post pandemia COVID-19. 2020. p. 1-54.

19. Morón-Araújo M. La Teleodontología una Herramienta Fundamental en Tiempos de Pandemia y post COVID -19, su Utilidad en las Diferentes Especialidades Odontológicas. Int J Odontostomatol [Internet]. 2021 Mar;15(1):43-50. Available from: http://www.scielo.cl/scielo.php?script=sci_arttext\&pid=S0718-381X20210 00100043\&lng=en\&nrm=iso\&tlng=en

20. Villegas Maestre JD, Ferrer Rosabal L de la C. Medidas de prevención y control de la COVID-19 en estomatología: "la nueva normalidad." Multimed [Internet]. 2021;25(2):e2060. Available from: https:// creativecommons.org/licenses/by-nc-sa/4.0/

21. Carrasco OV. Cómo escribir artículos de revisión. Rev Med La Paz [Internet]. 2009;15(1):63-9. Available from: http://www.scielo.org.bo/pdf/rmcmlp/v15n1/v15n1_a10.pdf

22. Morales Navarro D. Acciones del personal de salud del área estomatológica en relación a la COVID-19. Rev Cuba Estomatol2. 2020;57(1):e3245.

23. Ministerio de Salud. Situación actual COVID-19 Perú [Internet]. Gobierno del Perú. 2021. p. 1-5. Available 
from: https://covid19.minsa.gob.pe/sala_situacional.asp

24. Aylwin-Miranda I, Richaud-Hiza C, Moreno-Yañez Y. Guía para el Trabajo Clínico en Odontología Durante Pandemia por SARS-CoV-2, en el Hospital Clínico Dra. Eloísa Díaz. Int J Odontostomatol [Internet]. 2021 Mar;15(1):51-8. Available from: http://www.scielo.cl/scielo.php?script=sci_arttext\&pid=S0718-381X202 1000100051\&lng=en\&nrm=iso\&tlng=en

25. Fuentes R, Zaror C, Huanquilef M. Legislación y Normas Atingentes a la Atención Odontológica y el COVID-19. Una Perspectiva Desde las Clínicas Universitarias. Int J Odontostomatol [Internet]. 2020 Dec;14(4):481-8. Available from: http://www.scielo.cl/scielo.php?script=sci_arttext\&pid=S0718-381X20 $20000400481 \&$ Ing=en\&nrm=iso\&tlng=en

- Conflicto de intereses: La presente investigación no presenta conflicto de intereses entre los investigadores.

- Fuente de financiamiento: La presente investigación fue financiada por los investigadores. 